\title{
FAN1 mutations result in defective DNA damage repair and karyomegalic interstitial nephritis
}

$\mathrm{M}$

utations in FAN1 lead to a

defective DNA damage response (DDR) and karyomegalic interstitial nephritis (KIN), according to new research. "Our findings implicate susceptibility to environmental genotoxins and inadequate DNA repair as novel mechanisms contributing to renal fibrosis and [chronic kidney disease]", state the researchers.

To identify causative genes for nephronophthisis (NPHP)-like ciliopathies, Weibin Zhou, Edgar Otto et al. examined two affected siblings of New Zealand Maori descent. Renal histology demonstrated the presence of enlarged nuclei (karyomegaly), leading to a diagnosis of $\mathrm{KIN}-$ a rare NPHP-like fibrotic kidney disease. The researchers performed homozygosity mapping and exome sequencing, revealing a homozygous nonsense mutation in FAN1, which encodes Fanconi anemiaassociated nuclease 1 . Zhou et al. then sequenced FAN1 exons in DNA samples obtained from 10 families with KIN. They identified 12 different FAN1 mutations in nine of the 10 families; these mutations were absent in 96 healthy controls, thereby identifying recessive mutations in FAN1 as an important cause of KIN.

The FAN1 protein acts in a DNA repair pathway that is involved in the repair of interstrand cross-link (ICL) damage. To investigate the effect of the FAN1 mutation on genome maintenance and DDR, the researchers in collaboration with Agata Smogorzewska, studied the response of FAN1-mutant cells to the ICL-inducing agents mitomycin $\mathrm{C}$ and diepoxybutane. Upon exposure of primary fibroblasts and lymphoblastoid cell lines from individuals with FAN1 mutations to mitomycin C, chromatid breaks and radial chromosomes on metaphase spreads were observed, consistent with a role of FAN1 in DDR. Both mitomycin $\mathrm{C}$ and diepoxybutane reduced the survival of FAN1-mutant cells, demonstrating that FAN1 deficiency causes ICL sensitivity.

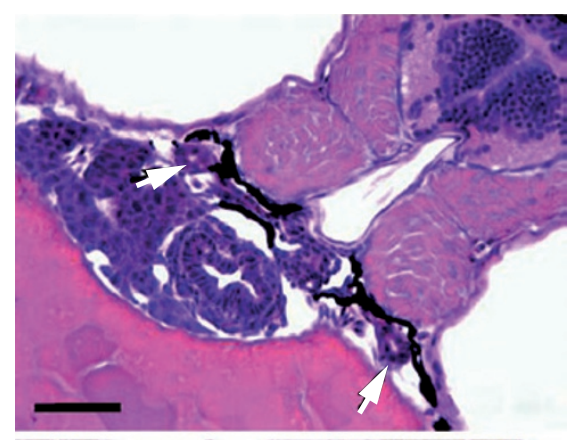

independently of the Fanconi anaemia pathway. In support of this theory, the researchers found that depletion of FANCD2 in FAN1-mutant cells resulted in a worsening of the mitomycin $C$ sensitivity defect, suggesting additive pathways.

Most DDR pathways and NPHP-like ciliopathies are conserved in zebrafish; therefore, the researchers performed morpholino oligonucleotide knockdown of $f a n 1$ in zebrafish embryos. Knockdown of fan 1 resulted in NPHP-like and DDR phenotypes, characterized by a shortened body axis, microcephaly, microphthalmia and massive apoptotic cell death. Zhou et al. found increased levels of $\gamma \mathrm{H} 2 \mathrm{AX}$, indicative of activated DDR signalling. Suppressing apoptosis by knocking down $\mathrm{p} 53$ function revealed phenotypes characteristic of NPHP-like ciliopathies, including pronephric renal cysts. "Loss of fan 1 function results in ciliopathy-related phenotypes, which are revealed when p53 function is inhibited", the authors state.

The researchers also examined $\gamma \mathrm{H} 2 \mathrm{AX}$ levels in a small sample of human biopsy samples from kidney transplant recipients. Levels of $\gamma \mathrm{H} 2 \mathrm{AX}$ were increased in samples with histological evidence of kidney damage, suggesting that DDR might contribute to renal damage in adults with chronic kidney disease as well as in individuals with NPHP-like ciliopathies.

Zhou et al. say that their study raises the question of whether individuals who carry hypomorphic alleles of FAN1 rather than two null alleles might develop renal failure later in life. "It will be important to examine a potential role for ICL-causing genotoxins in the absolute and relative increase in end-stage kidney disease", they explain.

Susan J. Allison

Original article Zhou, W. et al. FAN1 mutations cause karyomegalic interstitial nephritis, linking chronic kidney failure to defective DNA damage repair. Nat. Genet. doi:10.1038/ng.2347 